\title{
A TUTORIAL ON CONFORMAL GROUPS
}

\author{
IAN R. PORTEOUS \\ Department of Pure Mathematics, The University of Liverpool \\ PO Box 147, Liverpool L69 3BX, U.K. \\ E-mail: porteous@liverpool.ac.uk
}

\begin{abstract}
Our concern is with the group of conformal transformations of a finite-dimensional real quadratic space of signature $(p, q)$, that is one that is isomorphic to $\mathbb{R}^{p, q}$, the real vector space $\mathbb{R}^{p+q}$, furnished with the quadratic form

$$
x^{(2)}=x \cdot x=-x_{1}^{2}-x_{2}^{2}-\ldots-x_{p}^{2}+x_{p+1}^{2}+\ldots+x_{p+q}^{2},
$$

and especially with a description of this group that involves Clifford algebras.

1. Introduction. Let $X$ and $Y$ be finite-dimensional quadratic spaces and $f$ : $X \gg Y$ a smooth map, where the tail on the arrow indicates that the domain of definition of the map is an open subset of $X$ and not necessarily the whole of $X$. Then $f$ is said to be conformal if the differential $d f x$ of $f$ at any point $x$ is of the form $\rho(x) t$, where $\rho(x)$ is a non-zero real number and $t: X \rightarrow Y$ is an orthogonal map, that is, is such that for any $u, v \in X, d f x(u) \cdot d f x(v)=(\rho(x))^{2} u \cdot v$; that is it is conformal if it preserves angles. More generally, let $X$ and $Y$ be finite-dimensional smooth manifolds and $f: X \gg Y$ a smooth map. Then $f$ is said to be conformal if the differential $d f x$ of $f$ at any point $x$ of $X$ is a non-zero real multiple of an orthogonal map. It is well-known that any holomorphic map $f: \mathbb{C}>\mathbb{C}$, with $\mathbb{C}$ identified as a quadratic space with $\mathbb{R}^{2}$ with its standard scalar product, is conformal. Conformal transformations of quadratic spaces of dimension greater than 2 are more restricted, as follows, in the positive-definite case at least, from a theorem of Liouville (1850). It turns out that in studying such maps it is appropriate to compactify the quadratic spaces in question in a particular way that is known as the conformal compactification, this being quite distinct from the possibly more familiar projective compactification.
\end{abstract}

1991 Mathematics Subject Classification: Primary 46C20; Secondary 32G81.

The paper is in final form; although it would not be submitted for publication in any other journal, most of it is the basis of a fresh chapter in the author's current revision of Topological Geometry. 
2. Liouville's Theorem. The original Theorem of Liouville concerns smooth maps $f: \mathbb{R}^{3} \longrightarrow \mathbb{R}^{3}$, the vector space $\mathbb{R}^{3}$ being assigned its standard Euclidean metric. It follows from the standard theory of the curvature of a smooth surface in $\mathbb{R}^{3}$ that the parallels to such a surface together with the families of surfaces generated by the normals to either set of lines of curvature form a triply orthogonal set of families of surfaces. Dupin showed that the converse is true, in the sense that if one has such a triply orthogonal system then the surfaces of any two of the families cut out lines of curvature on the surfaces of the third family. Clearly a conformal transformation of $\mathbb{R}^{3}$ sends any such triply orthogonal system to another, so maps lines of curvature on any smooth surface to lines of curvature on the image surface. In particular it maps umbilical points of such a surface to umbilical points of the image surface. It then follows, by a theorem of Meusnier (1785), that the image by any conformal map of a sphere or plane is a sphere or plane. Such a map, by a theorem of Möbius, is representable as the composite of a finite number of orthogonal maps, translations or inversions of $\mathbb{R}^{3}$ in spheres, the simplest such inversion being inversion in the sphere, centre the origin, with unit radius, namely the map $\mathbb{R}^{3}>\mathbb{R}^{3}: x \mapsto x /|x|^{2}$. Moreover all such Möbius maps are conformal. This is the Theorem of Liouville.

The obvious analogue of this theorem then holds for positive-definite quadratic spaces of any finite dimension greater than 3 . The analogous statement for indefinite quadratic spaces also is true by a theorem of Haantjes (1938).

3. The projective compactification. Let $X$ be a finite-dimensional real vector space. Then any norm on $X$ induces the same topology. That is any subset of $X$ open with respect to any particular norm is also open with respect to any other norm.

Consider the map $X \rightarrow X \times \mathbb{R} ; x \mapsto(x, 1)$. This map is clearly injective but also induces an injective map

$$
X \rightarrow \mathcal{G}_{1}(X \times \mathbb{R}) ; x \mapsto[x, 1]=\mathbb{R}\{x, 1\}
$$

of $X$ to the projective space of lines through the origin in $X \times \mathbb{R}$. The projective space inherits the quotient topology from the topology of the linear space $X \times \mathbb{R}$ and is compact. The complement in the projective space of the image of the original space $X$ is by definition the hyperplane at infinity of $X$.

4. The conformal compactification. For a real non-degenerate quadratic space $X$ of signature $(p, q)$ there is an alternative compactification that often offers advantages. This is the conformal compactification defined as follows (cf. É. Cartan (1947), (1949), Kuiper (1949), Hermann (1979)).

For simplicity let the scalar product on $X$ be denoted by ·, and consider the injective map

$$
\iota: X \rightarrow X^{\prime \prime}=X \times \mathbb{R} \times \mathbb{R} ; x \mapsto(x, 1, x \cdot x)=(x, u, v) .
$$

This is not linear, but the image of $X$ is a subset of the quadric cone $Q$ in $X^{\prime \prime}$ with equation

$$
x \cdot x-u v=0 .
$$

The map then induces an injective map to the quadric in the projective space $\mathcal{G}_{1}\left(X^{\prime \prime}\right)$ 
with this homogeneous equation. This quadric is compact, being a closed subset of a compact space, and is defined to be the conformal compactification $\widehat{X}$ of the quadratic space $X$.

The quadric $\widehat{X}$ is easily shown to be homeomorphic to $S^{p} \times S^{q} / S^{0}$, where $S^{0}=\{1,-1\}$ acts on $S^{p} \times S^{q}$ by $(-1)(x, y)=(-x,-y)$. In particular, in the case that $p=0, q=n$ the quadric is homeomorphic to $S^{n}$ and in that case is a one-point compactification of $\mathbb{R}^{n}$.

Let $X^{\prime \prime}$ be assigned the quadratic form

$$
\left.(x, u, v) \mapsto x \cdot x-u v=x \cdot x-\left(\frac{1}{2}(u+v)\right)^{2}+\frac{1}{2}(u-v)\right)^{2},
$$

this being of signature $(p+1, q+1)$. The central result is then the corollary of the following:

Theorem 4.1. Let $X, X^{\prime \prime}$ and $Q$ be as above. Then

1. the map $\iota: X \rightarrow X^{\prime \prime} ; x \mapsto(x, x \cdot x, 1)$, with image a subset of $Q$, is an isometry;

2. the map $\pi: Q \gg X ;(x, u, v) \mapsto x / v$, defined where $x \cdot x=u v$ and $v \neq 0$, is conformal.

Pr o of. 1. The differential of $\iota$ at $x$ is the linear map

$$
d x \mapsto(d x, 2 x \cdot d x, 0),
$$

and $d x \cdot d x-(2 x \cdot d x)(0)=d x \cdot d x$.

2. The differential of $\pi$ at $(x, u, v)$ is the linear map

$$
(d x, d u, d v) \mapsto v^{-2}(v d x-x d v),
$$

with $x \cdot x=u v$ and $2 x \cdot d x=u d v+v d u$, implying that

$$
\begin{gathered}
(v d x-x d v) \cdot(v d x-x d v)=v^{2} d x \cdot d x-v d v(u d v+v d u)+u v d v^{2} \\
=v^{2}(d x \cdot d x-d u d v),
\end{gathered}
$$

so that $v^{-2}(v d x-x d v) \cdot v^{-2}(v d x-x d v)=v^{-2}(d x \cdot d x-d u d v)$.

Corollary 4.2. Let $t: X^{\prime \prime} \rightarrow X^{\prime \prime}$ be any orthogonal transformation of $X^{\prime \prime}$. Then the map $f=\pi t \iota: X \gg X$ is conformal.

From their form it is clear that such maps map conformal spheres (that is, quasispheres or hyperplanes) to conformal spheres, a quasi-sphere in the quadratic space $X$ being a submanifold of $X$ defined by an equation of the form $a x \cdot x+b \cdot x+c=0$, where $a, c \in \mathbb{R}$ and $b \in X, a$ and $b$ not both being zero, this being a genuine sphere in the case that the quadratic form on $X$ is positive definite and $a \neq 0$ and a plane in the case that $a=0$. It is a consequence of Liouville's theorem that, for $\operatorname{dim} X \geq 3$, all conformal maps $X \gg X$ are so induced. Clearly any such map $f$ extends to a map $\widehat{f}: \widehat{X} \rightarrow \widehat{X}$, with domain the whole of $\widehat{X}$.

It is clear in Corollary 4.2 that both $t$ and $-t$ induce the same conformal transformation of $X$. The conformal group $\operatorname{Con} f(X)$ is accordingly defined to be the quotient group $O\left(X^{\prime \prime}\right) / S^{0}$. Since the signature $(p+1, q+1)$ of $X^{\prime \prime}$ is indefinite, the group $O\left(X^{\prime \prime}\right)$ has four components. So $\operatorname{Con} f(X)$ has four or two, according to where the element $-1_{X^{\prime \prime}}$ lies. If it lies in the connected component of the identity, which is the case when $p$ and $q$ are both 
odd, implying that $p+1$ and $q+1$ are both even, then $\operatorname{Con} f(X)$ has four components, but if not, it has only two. The connected component of the identity is known as the Möbius group of $X$, denoted by $M(X)$.

We have already noted that $x \cdot x-u v=x \cdot x+\left(\frac{1}{2}(u-v)\right)^{2}-\left(\frac{1}{2}(u+v)\right)^{2}$. With this in mind the most usual chart to employ on the projective space $\mathcal{G}_{1}\left(X^{\prime \prime}\right)$ is the map

$$
[x, u, v] \mapsto\left(\frac{2 x}{u+v}, \frac{u-v}{u+v}\right) .
$$

Then the composite of the embedding of $X$ in $X^{\prime \prime}$ with the projection

$$
X^{\prime \prime}>X \times \mathbb{R} ;(x, u, v) \mapsto\left(\frac{2 x}{u+v}, \frac{u-v}{u+v}\right)
$$

is conformal, the product space $X \times \mathbb{R}$ being assigned the quadratic form $(x, w) \mapsto$ $x \cdot x+w^{2}$, since its differential is

$$
d x \mapsto\left(\frac{2 d x}{x \cdot x+1}-\frac{4 x x \cdot d x}{(x \cdot x+1)^{2}}, \frac{2 x \cdot d x}{x \cdot x+1}-\frac{2 x \cdot d x(x \cdot x-1)}{(x \cdot x+1)^{2}}\right),
$$

the quadratic norm of the image being

$$
\begin{gathered}
\frac{4}{(x \cdot x+1)^{4}}\left(((x \cdot x+1) d x-2 x x \cdot d x) \cdot((x \cdot x+1) d x-2 x x \cdot d x)+4(x \cdot d x)^{2}\right) \\
=\frac{4}{(x \cdot x+1)^{2}} d x \cdot d x .
\end{gathered}
$$

To clarify all this let us look at some simple examples.

EXAMPLE 4.3. Let $X=\mathbb{R}^{2}$ with its standard positive-definite scalar product. That is $X=\mathbb{R}^{0,2}$. Then the image of the map

$$
\mathbb{R}^{2} \rightarrow \mathcal{G}_{1}\left(\mathbb{R}^{4}\right) ;(x, y) \mapsto\left[x, y, 1, x^{2}+y^{2}\right],
$$

lies in the quadric with equation $x^{2}+y^{2}-u v=0$, this quadric being the conformal compactification of $X$.

Suppose that we make a change of variables to express the equation of the quadric as a sum of squares, an appropriate such choice being $x=x, y=y, z=\frac{1}{2}(u-v), t=\frac{1}{2}(u+v)$. Then the equation reduces to $x^{2}+y^{2}+z^{2}-t^{2}$ and the image lies entirely in the affine chart given by $t=1$, the map to this chart being the map

$$
\mathbb{R}^{2} \mapsto \mathbb{R}^{3} ;(x, y) \mapsto\left(\frac{2 x}{1+x^{2}+y^{2}}, \frac{2 y}{1+x^{2}+y^{2}}, \frac{1-x^{2}-y^{2}}{1+x^{2}+y^{2}}\right),
$$

with image a subset of the unit sphere $S^{2}$ in $\mathbb{R}^{3}$. Indeed the image is whole of this sphere with the exception of one point, the South Pole, $(0,0,-1)$.

This compactification, being a one-point compactification, is quite distinct from the projective compactification of the previous section. It is often presented in inverse form as the stereographic projection of the sphere, minus its South Pole, to its equatorial plane. Indeed the three points

$$
\left(\frac{2 x}{1+x^{2}+y^{2}}, \frac{2 y}{1+x^{2}+y^{2}}, \frac{1-x^{2}-y^{2}}{1+x^{2}+y^{2}}\right)
$$

of the sphere, $(x, y, 0)$ of the equatorial plane and $(0,0,-1)$, the South Pole, are collinear, as is readily verified. 
The inverse map is the map

$$
S^{2} \backslash(0,0,-1) \rightarrow \mathbb{R}^{2} ;\left(x^{\prime}, y^{\prime}, z^{\prime}\right) \mapsto\left(\frac{x^{\prime}}{1+z^{\prime}}, \frac{y^{\prime}}{1+z^{\prime}}\right) .
$$

ExAmPLE 4.4. Let $X=\mathbb{R}^{1,1}$. Then the image of the map

$$
\mathbb{R}^{2} \rightarrow \mathcal{G}_{1}\left(\mathbb{R}^{4}\right) ;(x, y) \mapsto\left[x, y, 1,-x^{2}+y^{2}\right],
$$

lies in the quadric with equation $-x^{2}+y^{2}-u v=0$, this quadric being the conformal compactification of $X$.

Suppose that we make the same change of variables as in Example 4.4 to express the equation of the quadric as a sum of squares, that is $x=x, y=y, z=\frac{1}{2}(u-v), t=$ $\frac{1}{2}(u+v)$. Then the equation reduces to $-x^{2}+y^{2}+z^{2}-t^{2}$. The image no longer lies entirely in any affine chart, but the map to the chart with $t=1$ is the map

$$
\mathbb{R}^{2}>\mathbb{R}^{3} ;(x, y) \mapsto\left(\frac{2 x}{1-x^{2}+y^{2}}, \frac{2 y}{1-x^{2}+y^{2}}, \frac{1+x^{2}-y^{2}}{1-x^{2}+y^{2}}\right),
$$

with domain the complement of the hyperbola with equation $x^{2}-y^{2}=1$ and image a subset of the hyperboloid of one sheet with equation $-X^{2}+Y^{2}+Z^{2}=1$.

5. Clifford algebras. It may be shown that, for any finite-dimensional real quadratic space $X$, there is a real associative algebra, $A$ say, with unit element 1 , containing isomorphic copies of $\mathbb{R}$ and $X$ as linear subspaces in such a way that, for all $x \in X, x^{2}=-x^{(2)}$.

If the algebra $A$ is generated as a ring by the copies of the scalars $\mathbb{R}$ and vectors $X$ or, equivalently, as a real algebra by $\{1\}$ and $X$, then $A$ is said to be a (real) Clifford algebra for $X$ (Clifford's term (1876) was geometric algebra).

To simplify notations in the above definition, $\mathbb{R}$ and $X$ have been identified with their copies in $A$. More strictly, there are linear injections $\alpha: \mathbb{R} \rightarrow A$ and $\beta: X \rightarrow A$ such that, for all $x \in X$,

$$
(\beta(x))^{2}=-\alpha\left(x^{(2)}\right),
$$

the unit element in $A$ being $\alpha(1)$.

The algebra $A$ is said to be a universal Clifford algebra for $X$ if it is generated as a real algebra by $X$ but by no linear subspace of $X$.

For example the algebra of quaternions $\mathbb{H}$ is a Clifford algebra for the real quadratic space $\mathbb{R}^{0,3}$ identified with the space of pure quaternions, but it is not a universal Clifford algebra for $\mathbb{R}^{0,3}$, since the algebra is generated by the subspace spanned by $\{i, j\}$. It is however a universal Clifford algebra for $\mathbb{R}^{0,2}$.

One of the characteristic properties of a Clifford algebra may be expressed in terms of an orthonormal basis as follows.

Proposition 5.1. Let $X$ be a finite-dimensional real quadratic space with an orthonormal basis $\left\{e_{i}: 0 \leq i<n\right\}$, where $n=\operatorname{dim} X$, and let $A$ be a real associative algebra with unit element 1 containing $\mathbb{R}$ and $X$ as linear subspaces. Then $x^{2}=-x^{(2)}$, for all $x \in X$, if and only if

$$
\begin{gathered}
e_{i}^{2}=-e_{i}^{(2)}, \text { for all } i \\
\text { and } e_{i} e_{j}+e_{j} e_{i}=0 \text { for all distinct } i \text { and } j
\end{gathered}
$$


This prompts the following definition.

An orthonormal subset of a real associative algebra $A$ with unit element 1 is a linearly independent subset $S$ of mutually anti-commuting elements of $A$, the square $a^{2}$ of any element $a \in S$ being 0,1 or -1 .

Proposition 5.2. Let $S$ be a subset of mutually anti-commuting elements of the algebra $A$ such that the square $a^{2}$ of any element $a \in S$ is 1 or -1 . Then $S$ is an orthonormal subset in $A$.

Proof. All that has to be verified is the linear independence of $S$.

An orthonormal subset $S$ none of whose elements has square zero, as in Proposition 5.2 , is said to be non-degenerate. If $p$ of the elements of $S$ have square +1 and if the remaining $q$ have square -1 , then $S$ is said to be of type $(p, q)$.

Any two universal Clifford algebras for the quadratic space $\mathbb{R}^{p, q}$ are naturally isomorphic, and any such will be denoted by $\mathbb{R}_{p, q}$. For example $\mathbb{R}_{1,1} \cong \mathbb{R}(2)$, the algebra of $2 \times 2$ matrices with entries in $\mathbb{R}$, a suitable orthonormal subset consisting of the matrices $\left(\begin{array}{ll}0 & 1 \\ 1 & 0\end{array}\right)$ and $\left(\begin{array}{cc}0 & -1 \\ 1 & 0\end{array}\right)$

In a universal Clifford algebra $A$ for a real quadratic space $X$ the space $\mathbb{R} \oplus X$, assigned the quadratic form

$$
(\lambda \oplus x)^{(2)}=(\lambda+x)(\lambda-x)
$$

is called the space of paravectors of $A$. Later it will be denoted by $Z$, with $z=\lambda+x$.

Any linear isomorphism $t: X \rightarrow X$ extends in a natural way both to an algebra isomorphism and to an algebra anti-automorphism of a universal Clifford algebra for $X$. In particular the identity on $X$ extends to an anti-involution of $A, A \rightarrow A ; g \mapsto \tilde{g}$, known as reversion, while the map $-1_{X}: x \mapsto-x$, extends both to an involution of $A$, $A \rightarrow A ; g \mapsto \widehat{g}$, known as the main or grade involution of $A$ and to an anti-involution $A \rightarrow A ; g \mapsto g^{-}$known as conjugation.

The elements of $A$ left invariant by the grade involution form a subalgebra $A^{0}$ of $A$, known as the even Clifford algebra for the quadratic space $X$. It can be proved that for any $(p, q)$ the algebra $\mathbb{R}_{p, q+1}^{0}$ is naturally isomorphic to $\mathbb{R}_{p, q}$.

The first stage in the construction of Clifford algebras is to show how to construct the universal Clifford algebra $\mathbb{R}_{p+1, q+1}$ for $\mathbb{R}^{p+1 . q+1}$, given $\mathbb{R}_{p, q}$, the universal algebra for $\mathbb{R}^{p, q}$. This is provided by the next proposition.

Proposition 5.3. Let $A$ be the algebra of $m \times m$ matrices over the field $\mathbb{K}$ or double field ${ }^{2} \mathbb{K}$ where $\mathbb{K}=\mathbb{R}, \mathbb{C}$ or $\mathbb{H}$, let $A(2)$ be the algebra of $2 \times 2$ matrices with entries in $A$ and let $S$ be an orthonormal subset of $A$ of type $(p, q)$, generating $A$ as a real algebra. Then the set of matrices

$$
\left\{\left(\begin{array}{cc}
a & 0 \\
0 & -a
\end{array}\right): a \in S\right\} \cup\left\{\left(\begin{array}{ll}
0 & 1 \\
1 & 0
\end{array}\right),\left(\begin{array}{cc}
0 & -1 \\
1 & 0
\end{array}\right)\right\}
$$

is an orthonormal subset of $A(2)$ of type $(p+1, q+1)$, generating $A(2)$ as a real algebra. 
Universal Clifford algebras $\mathbb{R}_{p, q}$ for each $p, q$ are given in the following table:

\begin{tabular}{|c|c|c|c|c|c|c|c|c|}
\hline & $q \rightarrow$ & & & & & & & \\
\hline \pm 1 & $\mathbb{R}$ & $\mathbb{C}$ & $\mathbb{H}$ & ${ }^{2} \mathbb{H}$ & $\mathbb{H}(2)$ & $\mathbb{C}(4)$ & $\mathbb{R}(8)$ & ${ }^{2} \mathbb{R}(8)$ \\
\hline $\mathbb{R}$ & ${ }^{2} \mathbb{R}$ & $\mathbb{R}(2)$ & $\mathbb{C}(2)$ & $\mathbb{H}(2)$ & ${ }^{2} \mathbb{H}(2)$ & $\mathbb{H}(4)$ & $\mathbb{C}(8)$ & $\mathbb{R}(16)$ \\
\hline $\mathbb{C}$ & $\mathbb{R}(2)$ & ${ }^{2} \mathbb{R}(2)$ & $\mathbb{R}(4)$ & $\mathbb{C}(4)$ & $\mathbb{H}(4)$ & ${ }^{2} \mathbb{H}(4)$ & $\mathbb{H}(8)$ & $\mathbb{C}(16)$ \\
\hline $\mathbb{H}$ & $\mathbb{C}(2)$ & $\mathbb{R}(4)$ & ${ }^{2} \mathbb{R}(4)$ & $\mathbb{R}(8)$ & $\mathbb{C}(8)$ & $\mathbb{H}(8)$ & ${ }^{2} \mathbb{H}(8)$ & $\mathbb{H}(16)$ \\
\hline${ }^{2} \mathbb{H}$ & $\mathbb{H}(2)$ & $\mathbb{C}(4)$ & $\mathbb{R}(8)$ & ${ }^{2} \mathbb{R}(8)$ & $\mathbb{R}(16)$ & $\mathbb{C}(16)$ & $\mathbb{H}(16)$ & ${ }^{2} \mathbb{H}(16)$ \\
\hline $\mathbb{H}(2)$ & ${ }^{2} \mathbb{H}(2)$ & $\mathbb{H}(4)$ & $\mathbb{C}(8)$ & $\mathbb{R}(16)$ & ${ }^{2} \mathbb{R}(16)$ & $\mathbb{R}(32)$ & $\mathbb{C}(32)$ & $\mathbb{H}(32)$ \\
\hline $\mathbb{C}(4)$ & $\mathbb{H}(4)$ & ${ }^{2} \mathbb{H}(4)$ & $\mathbb{H}(8)$ & $\mathbb{C}(16)$ & $\mathbb{R}(32)$ & ${ }^{2} \mathbb{R}(32)$ & $\mathbb{R}(64)$ & $\mathbb{C}(64)$ \\
\hline $\mathbb{R}(8)$ & $\mathbb{C}(8)$ & $\mathbb{H}(8)$ & ${ }^{2} \mathbb{H}(8)$ & $\mathbb{H}(16)$ & $\mathbb{C}(32)$ & $\mathbb{R}(64)$ & ${ }^{2} \mathbb{R}(64)$ & $\mathbb{R}(128)$ \\
\hline
\end{tabular}

The table of even algebras is obtained from this table by moving one space to the left and incorporating the additional column on the left-hand side. For any $(p, q)$ the even Clifford algebras $\mathbb{R}_{p, q}^{0}$ and $\mathbb{R}_{q, p}^{0}$ are naturally isomorphic.

The next two propositions give useful information on reversion and conjugation on the Clifford algebra $A(2)$ given these anti-involutions on $A$.

Proposition 5.4. Let $A$ be a universal Clifford algebra for a finite-dimensional real quadratic space $X$, of type $(p, q)$ and let $A(2)$ be the universal Clifford algebra for $X \times$ $\mathbb{R}^{1,1}$ constructed as in Proposition 5.3. Then the paravectors in $A(2)$ are of the form $\left(\begin{array}{cc}\lambda+x & v \\ u & (\lambda+x)^{-}\end{array}\right)$, where $\lambda+x$ is a paravector in $X$ with $\lambda, u, v \in \mathbb{R}$ and $x \in X$, and $(\lambda+x)^{-}=\lambda-x$.

Proposition 5.5. Let $A$ be a universal Clifford algebra for a finite-dimensional real quadratic space $X$, of type $(p, q)$ and let $A(2)$ be the universal Clifford algebra for $X \times \mathbb{R}^{1,1}$ constructed as in Proposition 5.3. Then

$$
\left(\begin{array}{ll}
a & c \\
b & d
\end{array}\right)^{-}=\left(\begin{array}{cc}
d^{\sim} & -c^{\sim} \\
-b^{\sim} & a^{\sim}
\end{array}\right) \text { and }\left(\begin{array}{ll}
a & c \\
b & d
\end{array}\right)^{\sim}=\left(\begin{array}{ll}
d^{-} & c^{-} \\
b^{-} & a^{-}
\end{array}\right)
$$

Proof. These are clearly true for vectors in $A(2)$ and so true for the whole of $A(2)$.

6. Rotations of $\mathbb{R}^{p, q}$. Clifford algebras permit simple descriptions of rotations of quadratic spaces. A rotation of a non-degenerate finite-dimensional real quadratic space $X$ is an isomorphism of $X$ that respects the orientations of $X$. Any such is the composite of an even number of linear hyperplane reflections of $X$, a linear hyperplane reflection of $X$ being a linear involution of $X$ that leaves invariant the vectors of the hyperplane, but cnanges the sign of vectors orthogonal to the hyperplane. Explicitly, let $A$ be a universal Clifford algebra for $S$ and let $a$ be any element of $X$ with $a \cdot a= \pm 1$. Then, for any $x \in X$, $a x a \in X$ and the map $X \rightarrow X ; x \mapsto-a x a$ is reflection in the hyperplane orthogonal to a. The Clifford group $\Gamma(X)$ consists of all $g \in A$ such that, for all $x \in X, g x \widehat{g}^{-1} \in X$, the map $X \rightarrow X ; x \mapsto g x \widehat{g}^{-1}$ then being an orthogonal automorphism of $X$, which is a rotation of $X$ if and only if $g \in A^{0}$, and any orthogonal automorphism of $X$ may be so induced. Indeed any element of $\Gamma$ may be shown to be expressible as the product of a finite number of invertible elements of $X$, this number being even when $g \in A^{0}$. The subgroup of even elements of $\Gamma$ is denoted by $\Gamma^{0}$. The quadratic norm of any element $g$ of $\Gamma$ is defined to be $N(g)=g^{-} g$, this being a non-zero real number. Moreover, for 
any $g \in \Gamma$ with $N(g)=1$ we have $\widehat{g}^{-1}=g^{\sim}$. The subgroup $\{g \in \Gamma ; N(g)= \pm 1\}$ of $\Gamma$ is defined to be $\operatorname{Spin} X$, and the subgroup $\{g \in \Gamma ; N(g)=1\}$ of $\Gamma$ is defined to be $\operatorname{Spin}^{+} X$. For a non-degenerate real quadratic space $X$ of index $(0, q)$ it turns out that $N(g)=1$ for all $g \in \Gamma$ so that $\operatorname{Spin}^{+} X=\operatorname{Spin} X$. There are short exact sequences of group homomorphisms:

$$
\{1\} \rightarrow S^{0} \rightarrow \operatorname{Spin} X \rightarrow S O(X) \rightarrow\{1\}
$$

and

$$
\{1\} \rightarrow S^{0} \rightarrow \operatorname{Spin}^{+} X \rightarrow S O^{+}(X) \rightarrow\{1\},
$$

where $S^{0}=\{1,-1\}$, the group $S O(X)$ being the group of rotations of $X$ and the group $S O^{+}(X)$ the subgroup that preserves the semi-orientations of $X$ in the case that the quadratic form on $X$ is of type $(p, q)$ with both $p$ and $q$ non-zero.

Let $A$ be a universal Clifford algebra for a non-degenerate real quadratic space $X$, and let $g$ be an invertible element of $A$ such that for any paravector $\lambda \oplus x$ of $A, g(\lambda \oplus x) \widehat{g}^{-1}$ also is a paravector of $A$. Then the map

$$
\mathbb{R} \oplus X \rightarrow \mathbb{R} \oplus X ; \lambda \oplus x \mapsto g(\lambda \oplus x) \widehat{g}^{-1}
$$

is a rotation of the quadratic space $Z$ of paravectors $\mathbb{R} \oplus X$, and any rotation of $Z$ may be so induced. That is, the group of such elements is isomorphic to $\Gamma^{0}(\mathbb{R} \oplus X)$.

Proposition 6.1. Let $A$ be a universal Clifford algebra for a positive-definite nondegenerate real quadratic space $X$, with Clifford group $\Gamma$ and let $\Gamma(2)$ denote the Clifford group of the Clifford algebra A(2). Then, for any

$$
\left(\begin{array}{ll}
a & c \\
b & d
\end{array}\right) \in \Gamma(2),
$$

1. either the elements of the main diagonal belong to $\Gamma^{0} \cup\{0\}$ and those of the other diagonal to $\Gamma^{1} \cup\{0\}$, or vice versa;

2. $a d^{\sim}-c b^{\sim}$ is real and non-zero;

3. $a b^{-} \in X$ and $c d^{-} \in X$, or equivalently $a b^{\sim} \in X$ and $c d^{\sim} \in X$, since, for any $g \in \Gamma, g^{\sim}= \pm g^{-}$.

Pr o of. Part 1 is by induction on the number of factors in some representation of the matrix as a product of vectors. Part 2 follows from the fact that $a d^{\sim}-c b^{\sim}$ is just the quadratic norm of the matrix. Part 3 follows at once by letting the matrix act on each of the vectors $\left(\begin{array}{ll}0 & 1 \\ 0 & 0\end{array}\right)$ and $\left(\begin{array}{ll}0 & 0 \\ 1 & 0\end{array}\right)$.

That this is a characterisation of the elements of $\Gamma(2)$ in the case that the quadratic space $X$ is positive-definite will be indicated later. In the case of an indefinite space $X$ the result is false, as is at once clear, since it is false for a vector $\left(\begin{array}{cc}x & v \\ u & -x\end{array}\right)$ for which $x \neq 0$ but $x \cdot x=0$.

In fact it can even be the case that none of the entries in the $2 \times 2$ matrix representing an element of $\Gamma(2)$ is invertible, as an example of Maks (1989), (1992) shows. Consider generators $e_{1}$ and $e_{2}$ of $\mathbb{R}^{1,1}$ with $e_{1}^{2}=-1$ and $e_{2}^{2}=1$, and take the standard model of 
$\mathbb{R}^{2,2}$ in $\mathbb{R}_{1,1}(2)$. Then the rotation of $\mathbb{R}^{2,2}$ that sends $e_{1}$ to $\left(\begin{array}{cc}0 & -1 \\ 1 & 0\end{array}\right)$, and that vector to $-e_{1}$, and similarly sends $e_{2}$ to $\left(\begin{array}{ll}0 & 1 \\ 1 & 0\end{array}\right)$, and that vector to $-e_{2}$, is induced by the matrix

$$
\left(\begin{array}{cc}
1 & e_{1} \\
e_{1} & 1
\end{array}\right)\left(\begin{array}{cc}
1 & -e_{2} \\
e_{2} & 1
\end{array}\right)=\left(\begin{array}{cc}
1+e_{1} e_{2} & e_{1}-e_{2} \\
e_{1}+e_{2} & 1-e_{1} e_{2}
\end{array}\right)
$$

of $\Gamma(2)$. None of the entries in this matrix are invertible elements of $\mathbb{R}_{1,1}$.

The groups $\operatorname{Spin}(n)$ for $n \leq 6$, and the groups $\operatorname{Spin}^{+}(p, q)$ for $p+q \leq 6$, in the case that both $p$ and $q$ are non-zero, are shown in Table 2 .

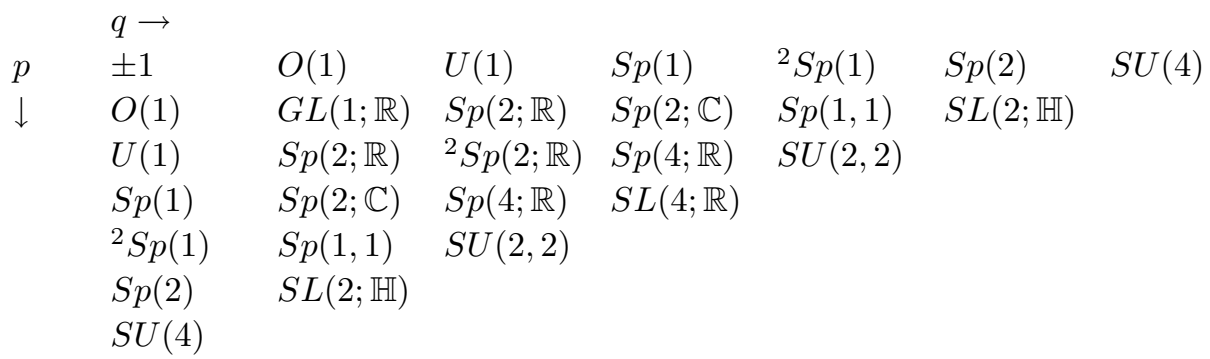

The notations are mostly standard. For example $S p(4 ; \mathbb{R})$ denotes the symplectic group of $4 \times 4$ real matrices that preserve the skew-symmetric product

$$
\mathbb{R}^{4} \times \mathbb{R}^{4} \rightarrow \mathbb{R}^{4} ;\left(\left(x_{1}, x_{2}, x_{3}, x_{4}\right),\left(y_{1}, y_{2}, y_{3}, y_{4}\right)\right) \rightarrow x_{1} y_{2}-x_{2} y_{1}+x_{3} y_{4}-x_{4} y_{3},
$$

while $S L(2 ; \mathbb{H})$ denotes the special linear group of all $2 \times 2$ matrices with entries in $\mathbb{H}$ and of determinant 1 , the determinant being defined to be the determinant of the matrix when represented as an element of $\mathbb{C}(4)$. Only those with $p$ or $q$ zero are compact.

For more details on Clifford algebras, and Table 2 in particular, the reader is referred to Porteous (1981) and (1993).

7. Möbius transformations of $\mathbb{R}^{2}$. The appropriate Clifford algebra in which to study rotations of the Euclidean plane $\mathbb{R}^{2}$ is the algebra $\mathbb{R}_{0,2}^{0}$ of complex numbers $\mathbb{C}$. The plane itself is represented by the plane of paravectors which in this case is the whole of $\mathbb{C}$. Now the conformal compactification sits naturally inside the Clifford algebra $\mathbb{R}_{1,3}^{0}$ which is representable by the matrix algebra $\mathbb{C}(2)$, the complex number $z$ then being represented by the matrix

that is explicitly by the element

$$
\left(\begin{array}{cc}
z & z \bar{z} \\
1 & \bar{z}
\end{array}\right)
$$

$$
x\left(\begin{array}{ll}
1 & 0 \\
0 & 1
\end{array}\right)+\mathrm{i} y\left(\begin{array}{cc}
1 & 0 \\
0 & -1
\end{array}\right)+\frac{1}{2}(1+z \bar{z})\left(\begin{array}{ll}
0 & 1 \\
1 & 0
\end{array}\right)+\frac{1}{2}(1-z \bar{z})\left(\begin{array}{cc}
0 & -1 \\
1 & 0
\end{array}\right) .
$$

More generally any element of $\mathbb{R}^{1,3}$ sitting as a paravector in $\mathbb{R}_{1,3}^{0}$ is represented by an element of $\mathbb{C}(2)$ of the form

$$
\left(\begin{array}{cc} 
& v \\
u & \bar{z}
\end{array}\right)
$$

where $z \in \mathbb{C}$ and $u$ and $v$ are real. 
Consider now an element of $\operatorname{Spin}^{+}(1,3)$ represented by an element of $\mathbb{C}(2)$ of the form

$$
\left(\begin{array}{ll}
a & c \\
b & d
\end{array}\right)
$$

This maps the above paravector to

$$
\left(\begin{array}{ll}
a & c \\
b & d
\end{array}\right)\left(\begin{array}{ll}
z & v \\
u & \bar{z}
\end{array}\right)\left(\begin{array}{ll}
a & c \\
b & d
\end{array}\right)^{\sim}=\left(\begin{array}{ll}
a & c \\
b & d
\end{array}\right)\left(\begin{array}{ll}
z & v \\
u & \bar{z}
\end{array}\right)\left(\begin{array}{ll}
\bar{d} & \bar{c} \\
\bar{b} & \bar{a}
\end{array}\right) .
$$

In particular it maps the paravector

$$
\left(\begin{array}{cc}
z & z \bar{z} \\
1 & \bar{z}
\end{array}\right)=\left(\begin{array}{l}
z \\
1
\end{array}\right)\left(\begin{array}{ll}
1 & \bar{z}
\end{array}\right)
$$

representing the complex number $z$ to

$$
\left(\begin{array}{ll}
a & c \\
b & d
\end{array}\right)\left(\begin{array}{cc}
z & z \bar{z} \\
1 & \bar{z}
\end{array}\right)\left(\begin{array}{cc}
\bar{d} & \bar{c} \\
\bar{b} & \bar{a}
\end{array}\right)=\left(\begin{array}{cc}
\lambda z^{\prime} & \lambda z^{\prime} \overline{z^{\prime}} \\
\lambda & \lambda \overline{z^{\prime}}
\end{array}\right)=\lambda\left(\begin{array}{cc}
z^{\prime} & z^{\prime} \overline{z^{\prime}} \\
1 & z^{\prime}
\end{array}\right)
$$

where $z^{\prime}=\frac{a z+c}{b z+d}$ and $\lambda$ is the real number $|b z+d|^{2}$.

Now the conformal compactification of $\mathbb{C}$ is a one-point compactification and it is natural to denote the additional point by $\infty$. One easily verifies that this point is represented in $\mathbb{C}(2)$ by the matrix

$$
\left(\begin{array}{ll}
0 & 1 \\
0 & 0
\end{array}\right)
$$

and that the image of this matrix by the above element of $\operatorname{Spin}^{+}(1,3)$ is the matrix

$$
\left(\begin{array}{cc}
\bar{b} & a \bar{a} \\
b \bar{b} & b \bar{a}
\end{array}\right)
$$

that represents the complex number $a / b$, when $b \neq 0$ and $\infty$ otherwise.

A map

$$
\mathbb{C} \cup\{\infty\} \rightarrow \mathbb{C} \cup\{\infty\}: z \mapsto \frac{a z+c}{b z+d},
$$

where $a, b, c, d \in \mathbb{C}$ and $a d-b c=1$, is known as a Möbius map. It represents a special conformal transformation of the conformal compactification of $\mathbb{R}^{0,2}$, namely one that respects the orientations of $\mathbb{R}^{0,2}$ and its compactification.

For such a Möbius map the induced map of $\mathbb{C}^{2}$ defined by the matrix

$$
\left(\begin{array}{ll}
a & c \\
b & d
\end{array}\right)
$$

will restrict to a rotation of this sphere if and only if this matrix lies in the copy of $\operatorname{Spin}^{+}(0,3)=\operatorname{Spin} 3$ naturally included in $\operatorname{Spin}^{+}(1,3)$. Such matrices are those that represent unit quaternions, namely those of the form

$$
\left(\begin{array}{cc}
a & -\bar{b} \\
b & \bar{a}
\end{array}\right) .
$$

ExAmple 7.1. There is a unique Möbius map of $\mathbb{C}$ to $\mathbb{C}$ that sends 0 to 1,1 to $\mathrm{i}$ and i to 0 . The induced rotation of the Riemann sphere is rotation of the sphere through an angle $\frac{2 \pi}{3}$ about the line with equations $x=y=z$. 
Proof. Let the Möbius map be

$$
z \mapsto \frac{a z+c}{b z+d}
$$

Then $\frac{c}{d}=1$, that is $c=d, \frac{a+c}{b+d}=i$, that is $a+c=(b+d) \mathrm{i}$, and $\frac{a \mathrm{i}+c}{b \mathrm{i}+d}=0$, that is $a \mathrm{i}+c=0$. Try $a=1$. Then $c=d=-\mathrm{i}$ and $1-i=b \mathrm{i}+1$ so that $b=-1$. This gives the map

$$
z \mapsto \frac{z-\mathrm{i}}{-z-\mathrm{i}}
$$

However then $a d-b c=1(-\mathrm{i})-(-1)(-\mathrm{i})=-2 \mathrm{i}=(1-\mathrm{i})^{2}$, and the inverse of $1-\mathrm{i}$ is $\frac{1}{2}(1+i)$. So finally the required map is

$$
z \mapsto \frac{\frac{1}{2}(1+i) z+\frac{1}{2}(1-i)}{-\frac{1}{2}(1+i) z+\frac{1}{2}(1-i)} .
$$

The fixed points of this map are given by $z-\mathrm{i}=(-z-\mathrm{i}) z$, that is by the quadratic equation $z^{2}+(1+\mathrm{i}) z-\mathrm{i}=0$, with $\operatorname{roots} z=\frac{1}{2}(-1-\mathrm{i} \pm \sqrt{6 i})$.

8. Möbius transformations of $\mathbb{R}^{p, q}$. The general case can be handled in exactly the same way as the case of $\mathbb{R}^{2}$ has been treated in the previous section. The appropriate Clifford algebra in which to study rotations of the quadratic space $\mathbb{R}^{p, q}$ is the algebra $\mathbb{R}_{p, q}^{0}$, isomorphic to the Clifford algebra $\mathbb{R}_{p, q-1}$. The quadratic space itself is then represented by, and will be identified with, the space of paravectors $Z$ in the algebra $\mathbb{R}_{p, q-1}$, with $z \cdot z=z z^{-}$, for any $z \in Z$. The conformal compactification sits naturally inside the algebra $\mathbb{R}_{p+1, q+1}^{0}$ which is isomorphic to the algebra $\mathbb{R}_{p+1, q} \cong \mathbb{R}_{p, q-1}(2)$ of $2 \times 2$ matrices with entries in $\mathbb{R}_{p, q-1}$.

The paravector $z$ is then represented by the matrix

$$
\left(\begin{array}{cc}
z & z z^{-} \\
1 & z^{-}
\end{array}\right)
$$

that is explicitly by the element

$$
\left(\begin{array}{cc}
z & 0 \\
0 & z^{-}
\end{array}\right)+\frac{1}{2}\left(1+z z^{-}\right)\left(\begin{array}{ll}
0 & 1 \\
1 & 0
\end{array}\right)+\frac{1}{2}\left(1-z z^{-}\right)\left(\begin{array}{cc}
0 & -1 \\
1 & 0
\end{array}\right) .
$$

More generally (cf. Proposition 5.4) any element of $\mathbb{R}^{p+1, q+1}$ is represented by a paravector in $\mathbb{R}_{p+1, q} \cong \mathbb{R}_{p, q-1}(2)$ of the form

$$
\left(\begin{array}{cc}
z & v \\
u & z^{-}
\end{array}\right)
$$

where $z$ is a paravector in $\mathbb{R}_{p, q-1}$ and $u$ and $v$ are real.

Consider now an element of $\operatorname{Spin}^{+}(p+1, q)$ represented by an element of the algebra $\mathbb{R}_{p, q-1}(2)$ of the form

$$
\left(\begin{array}{ll}
a & c \\
b & d
\end{array}\right) .
$$


This maps the above paravector to

$$
\left(\begin{array}{ll}
a & c \\
b & d
\end{array}\right)\left(\begin{array}{cc}
z & v \\
u & z^{-}
\end{array}\right)\left(\begin{array}{ll}
a & c \\
b & d
\end{array}\right)^{\sim}=\left(\begin{array}{ll}
a & c \\
b & d
\end{array}\right)\left(\begin{array}{cc}
z & v \\
u & z^{-}
\end{array}\right)\left(\begin{array}{ll}
d^{-} & c^{-} \\
b^{-} & a^{-}
\end{array}\right)
$$

by Proposition 5.5. In particular it maps the paravector

$$
\left(\begin{array}{cc}
z & z z^{-} \\
1 & z^{-}
\end{array}\right)=\left(\begin{array}{l}
z \\
1
\end{array}\right)\left(\begin{array}{ll}
1 & z^{-}
\end{array}\right)
$$

representing the paravector $z$ to

$$
\begin{aligned}
\left(\begin{array}{ll}
a & c \\
b & d
\end{array}\right)\left(\begin{array}{cc}
z & z z^{-} \\
1 & z^{-}
\end{array}\right)\left(\begin{array}{cc}
- & c^{-} \\
b^{-} & -
\end{array}\right) & =\left(\begin{array}{cc}
(a z+c)(b z+d)^{-} & (a z+c)(a z+c)^{-} \\
(b z+d)(b z+d)^{-} & (b z+d)(a z+c)^{-}
\end{array}\right) \\
& =\lambda\left(\begin{array}{cc}
z^{\prime} & z^{\prime} z^{\prime-} \\
1 & z^{\prime}
\end{array}\right),
\end{aligned}
$$

where $z^{\prime}=(a z+c)(b z+d)^{-1}$ and $\lambda$ is the real number $(b z+d)(b z+d)^{-}$.

The matrix $\left(\begin{array}{ll}a & c \\ b & d\end{array}\right)$ representing the induced special conformal transformation of the conformal compactification of $\mathbb{R}^{p, q}$ is known as a Vahlen representation (cf. Vahlen, (1902)), a special transformation being one that respects the orientation (and, in the case that $p$ and $q$ are both odd, the semi-orientations) of the compactification, this representation being unique up to sign in the case that $p q$ is even, but with a four-fold ambiguity in its definition in the case that $p q$ is odd.

For example the translation $x \mapsto x+c$ is represented by the matrix $\left(\begin{array}{ll}1 & c \\ 0 & 1\end{array}\right)$ and the inflation $x \mapsto \rho x$ by the positive scalar $\rho$ by the matrix $\left(\begin{array}{cc}\sqrt{\rho} & 0 \\ 0 & \sqrt{\rho}^{-1}\end{array}\right)$, while inversion in the unit quasi-sphere composed with the hyperplane reflection $x \mapsto-x^{-}$is represented by the matrix $\left(\begin{array}{cc}0 & -1 \\ 1 & 0\end{array}\right)$.

Any Möbius transformation is expressible as a product of a finite number of inversions in spheres and hyperplane reflections.

An important special case in which $p q$ is odd is that of $\mathbb{R}^{1,3}$ in which case the component of the identity of the conformal group is covered four times rather than twice by the group $S U(2,2)$, the identity transformation of the space being represented by each of the matrices

$$
\left(\begin{array}{ll}
1 & 0 \\
0 & 1
\end{array}\right), \quad\left(\begin{array}{cc}
-1 & 0 \\
0 & -1
\end{array}\right), \quad\left(\begin{array}{ll}
\mathrm{I} & 0 \\
0 & \mathrm{I}
\end{array}\right) \text { and }\left(\begin{array}{cc}
\mathrm{I} & 0 \\
0 & -\mathrm{I}
\end{array}\right),
$$

where $I$ is the $2 \times 2$ complex matrix $\left(\begin{array}{ll}\mathrm{i} & 0 \\ 0 & \mathrm{i}\end{array}\right)$.

The complete set of Möbius groups, for $p+q \leq 4$, is given in the following theorem.

TheOREM 8.1. Let $M_{p, q}$ denote the Möbius group $M\left(\mathbb{R}^{p, q}\right)$. Then

$$
\begin{array}{rlrl}
M_{0,1} \cong M_{1,0} \cong & \cong p(2, \mathbb{R}) / S^{0} & \\
M_{0,2} \cong M_{2,0} & \cong & S p(2, \mathbb{C}) / S^{0} \\
M_{1,1} & \cong{ }^{2}\left(S p(2 ; \mathbb{R}) / S^{0}\right) & (p q \text { odd }) & \\
M_{0,3} \cong M_{3,0} & \cong & S p(1,1) / S^{0}
\end{array}
$$




$$
\begin{array}{rlll}
M_{1,2} \cong M_{2,1} & \cong & & S p(4, \mathbb{R}) / S^{0} \\
M_{0,4} \cong M_{4,0} & \cong & & S L(2, \mathbb{H}) / S^{0} \\
M_{1,3} \cong M_{3,1} & \cong & & \\
\left.M_{2,2} \cong S L(2,2)\right) / C(4) & (p q \text { odd })
\end{array}
$$

Proof. These results follow directly from the remarks following Corollary 4.2 and Table 2 .

Finally we have the following converse to Proposition 6 .

Proposition 8.2. Let $A$ be a universal Clifford algebra for a positive-definite real quadratic space $X$, with Clifford group $\Gamma$, and let $\Gamma(2)$ denote the Clifford group of the Clifford algebra $A(2)$. Then conditions 1 to 3 of Proposition 6.1 characterise elements of $\Gamma(2)$.

Indication of Proof: What has to be proved is that for any matrix $\left(\begin{array}{ll}a & c \\ b & d\end{array}\right) \in A(2)$ satisfying these conditions and for any $\left(\begin{array}{cc}x & v \\ u & x^{-}\end{array}\right)$, where $x \in X$ and $u, v \in \mathbb{R}$, the matrix

$$
\left(\begin{array}{ll}
a & c \\
b & d
\end{array}\right)\left(\begin{array}{ll}
x & v \\
u & x^{-}
\end{array}\right)\left(\begin{array}{ll}
d^{-} & c^{-} \\
b^{-} & a^{-}
\end{array}\right)=\left(\begin{array}{ll}
x^{\prime} & v^{\prime} \\
u^{\prime} & x^{\prime-}
\end{array}\right)
$$

where $x^{\prime} \in X$ and $u^{\prime}$ and $v^{\prime} \in \mathbb{R}$. This is easily verified in the particular case that $x=0$, and the problem is then reducible to proving things in the case that $u=1$ and $v=x x^{-}$. This in its turn is reducible by Section 8 to proving that, for any $x \in X$, $(a x+c)(b x+d)^{-1} \in X$. For the details see Ahlfors (1985).

Proposition 8.2 remains true even in the indefinite case, provided that conditions $1-3$ are suitably amended. In particular the set $\Gamma \cup\{0\}$ has to be replaced by the monoid $\mathbf{T}$ of all products of a finite number of vectors, whether invertible or not. Let $\mathbf{T}^{0}$ denote the set of all products of an even number of vectors and $\mathbf{T}^{1}$ the set of all products of an odd number of vectors. Then the amended conditons (in a form essentially due to Cnops) are

1. either the elements of the main diagonal belong to $\mathbf{T}^{0}$ and those of the other diagonal to $\mathbf{T}^{1}$, or vice versa;

2. $a d^{\sim}-c b^{\sim}$ is real and non-zero;

3. $a b^{-}, c d^{-}, a^{-} c$ and $b^{-} d \in X$, or equivalently $a b^{\sim}, c d^{\sim}, a^{\sim} c$ and $b^{\sim} d \in X$, since, for any $g \in \mathbf{T}, g^{\sim}= \pm g^{-}$.

Since non-zero vectors need not be invertible the method of proof of Ahlfors does not generalise. For the details see Maks (1989), Fillmore and Springer (1990) and Cnops (1994).

Acknowledgment. I am greatly indebted to Pertti Lounesto for his encyclopaedic knowledge of the history of Clifford algebras, and such related topics as conformal groups, and for keeping me right on a number of points of detail. A very similar treatment of the conformal groups to that of this paper is to be found in the recent thesis of Jan Cnops (1994), and I am grateful to him for his comments. I am also grateful to Guy Laville for drawing my attention to the papers (1947) and (1949) (his last) of Élie Cartan. 


\section{References}

[1] L. Ahlfors, Möbius transformations and Clifford numbers, I. Chavel, H.M. Parkas (eds.). Differential Geometry and Complex Analysis. Dedicated to H.E. Rauch, Springer-Verlag, Berlin, (1985), 65-73.

[2] É. Cartan, Sur l'espace anallagmatique réel à $n$ dimensions, Ann. Polon. Math. 20 (1947), 266-278.

[3] —, Deux théorèmes de géométrie anallagmatique réelle à $n$ dimensions, Ann. Mat. Pura Appl. (4)28 (1949), 1-12.

[4] W.K. Clifford, (1876) On the Classification of Geometric Algebras, published as Paper XLIII in Mathematical papers. Edited by R. Tucker, Macmillan, London (1882).

[5] J. Cnops, Hurwitz Pairs and Applications of Möbius Transformations. Thesis, Universiteit Gent, 1994.

[6] J. Fillmore, and A. Springer, Möbius groups over general fields using Clifford algebras associated with spheres, Int. J. Theo. Phys. 29 (1990), 225-246

[7] J. Haantjes, Conformal representations of an $n$-dimensional euclidean space with a non-definite fundamental form on itself, Proc. Ned. Akad. Wet. (Math.) 40 (1937), 700-705.

[8] R. Hermann, Appendix Kleinian mathematics from an advanced standpoint, A: Conformal and non-Euclidean geometry in $R^{3}$ from the Kleinian viewpoint, bound with Klein F. Developments of Mathematics in the 19th century. Translated by M. Ackerman, Math. Sci. Press, Brookline, Mass. USA, 1979, 367-376.

[9] N.H. Kuiper, On conformally-flat spaces in the large, Ann. Math. 50 (1949), 916-924.

[10] J. Liouville, Appendix to Monge, G. Application de l'analyse à la geométrie, 5 éd. par Liouville, 1850.

[11] J. Maks, Modulo $(1,1)$ periodicity of Clifford algebras and the generalized (anti-)Möbius transformations. PhD Thesis, Technische Universiteit Delft., 1989.

[12] - Clifford algebras and Möbius transformations, in A. Micali et al. (eds.) Clifford Algebras and their Applications in Mathematical Physics, Kluwer Acad. Publ., Dordrecht 1992.

[13] J.-B.-M.-C. Meusnier, Mémoire sur la courbure des surfaces, Mémoire Div. Sav., 10 (1785), 477-510.

[14] I. R. Porteous, Topological Geometry, 2nd Edition, with additional material on Triality, Cambridge University Press, 1981. (The part of this book concerned with Clifford algebras forms part of a new edition entitled Clifford Algebras and the Classical Groups published in 1995 by Cambridge University Press.)

[15] -, Clifford algebra tables in F. Brackx et al (eds.). Clifford Algebras and their applications in Mathematical Physics, Kluwer Academic Publishers, 1993, 13-22.

[16] K. Th. Vahlen, Über Bewegungen und complexe Zahlen, Math. Ann. 55 (1902), 585593. 